\title{
ZNF280A promotes lung adenocarcinoma development by regulating the expression of EIF3C
}

\author{
Hongsheng Liu', Yingzhi Qin ${ }^{1}$, Na Zhou², Dongjie Ma ${ }^{1}$ and Yingyi Wang ${ }^{2}$
}

\begin{abstract}
Lung adenocarcinoma (LUAD) is the most common histological subtype in non-small cell lung cancer, which is the malignant tumor with the highest mortality and morbidity in the world. Herein, ZNF280A, a member of the zinc finger protein family carrying two consecutive Cys2His2 zinc finger domains, was shown by us to act as a tumor driver in LUAD. The immunohistochemical analysis of ZNF280A in LUAD indicated its positive correlation with tumor grade, pathological stage and lymphatic metastasis, and negative relationship with patients' survival. A loss-of-function study revealed the inhibition of LUAD development by ZNF280A in vitro and in vivo, whereas ZNF280A overexpression induced opposite effects. Statistical analysis of gene expression profiling in LUAD cells with or without ZNF280A knockdown identified EIF3C as a potential downstream of ZNF280A, which possesses similar regulatory effects on phenotypes of LUAD cells with ZNF280A. Moreover, downregulation of EIF3C in ZNF280A-overexpressed cells could attenuate neutralize the ZNF280A-induced promotion of LUAD. In summary, our study demonstrated that ZNF280A may promote the development of LUAD by regulating cell proliferation, apoptosis, cell cycle, and cell migration and probably via interacting EIF3C.
\end{abstract}

\section{Introduction}

Lung cancer is one of the malignant tumors that seriously threaten human health and life. According to the statistics of American Cancer Society, the incidence and mortality of lung cancer rank first among all malignant tumors $^{1,2}$. According to histopathology, lung cancer can be divided into small cell lung cancer and non-small cell lung cancer (NSCLC). Among them, the total incidence of NSCLC is $\sim 80-85 \%$, which can be mainly divided into adenocarcinoma, squamous cell carcinoma, and large cell carcinoma, among which lung adenocarcinoma (LUAD) is the most common type of lung cancer ${ }^{3,4}$. Frequently, LUAD is surrounded by many blood vessels, thus prone to metastasis and invasion ${ }^{5}$. The early symptoms of lung cancer are not obvious, almost no discomfort, so the vast

\footnotetext{
Correspondence: Yingyi Wang (waltwyy@163.com)

${ }^{1}$ Department of Thoracic Surgery, Peking Union Medical College Hospital,

Beijing, China

${ }^{2}$ Department of Medical Oncology, Peking Union Medical College Hospital,

Beijing, China

These authors contributed equally: Hongsheng Liu, Yingzhi Qin

Edited by S. Tait
}

majority of patients suffered from advanced lung cancer at the time of diagnosis, in which the lesions have occurred local invasion or distal metastasis ${ }^{6}$. At present, the first choice and main means of treatment for lung cancer are still surgical treatment combined with radiotherapy or chemotherapy. In recent years, although molecular targeted therapy and immunotherapy have received great attention and developed rapidly, the prognostic improvement for LUAD patients is still not ideal? Therefore, exploring key regulatory molecules associated with tumor proliferation or migration is essential for understanding the molecular mechanisms of lung cancer and developing more efficient therapeutic strategies ${ }^{8-10}$.

Zinc finger proteins are a type of transcription factor with characteristic "finger" domains ${ }^{11}$. Zinc finger proteins are widely distributed in various eukaryotes, and play a function of regulating and controlling gene expression. The most significant structural feature of zinc finger protein family members is that they can generate short three-dimensional structural models of polypeptides according to their own folding patterns, and the

\section{(c) The Author(s) 2021}

(c) (i) Open Access This article is licensed under a Creative Commons Attribution 4.0 International License, which permits use, sharing, adaptation, distribution and reproduction c. in any medium or format, as long as you give appropriate credit to the original author(s) and the source, provide a link to the Creative Commons license, and indicate if changes were made. The images or other third party material in this article are included in the article's Creative Commons license, unless indicated otherwise in a credit line to the material. If material is not included in the article's Creative Commons license and your intended use is not permitted by statutory regulation or exceeds the permitted use, you will need to obtain permission directly from the copyright holder. To view a copy of this license, visit http://creativecommons.org/licenses/by/4.0/. 
combination with zinc ions can help them maintain the stability of this molecular structure ${ }^{11}$. The functional research on zinc finger proteins covers a wide range, and the existing evidence clearly points out that it has an important role in embryonic development, cell growth and differentiation, and signal transduction ${ }^{12}$. In recent years, there have been more and more studies on the regulatory role of zinc finger proteins in human tumors $^{12,13}$. Multiple zinc finger proteins have been found to have a role in promoting disease development in a variety of tumors. ZNF280A was first identified in the comprehensive high-resolution genome-wide analysis of mantle cell lymphoma ${ }^{14}$. It carries two consecutive Cys2His2 zinc finger domains and is a member of the zinc finger protein family. However, the relationship between ZNF280A and most types of human tumors including LUAD has yet to be developed.

In this study, we explored the biological function and downstream mechanism of ZNF280A in the development and progression of LUAD. Analysis of clinical specimens revealed the potential of ZNF280A as an indicator for tumor progression and poor prognosis of LUAD patients. Loss-of-function and gain-of-function studies revealed the regulatory role of ZNF280A in the development and progression of LUAD by influencing cell proliferation, colony formation, cell apoptosis, cell cycle distribution, and cell migration. The xenografts formed by inoculation of cells with ZNF280A knockdown progress much slower relative to the control group. Furthermore, EIF3C was screened as the potential downstream of ZNF280A to mediate the regulation of LUAD development. In a word, this study identified ZNF280A as an oncogene-like factor in the development of LUAD, which may be used as an effective therapeutic target in LUAD treatment.

\section{Results \\ ZNF280A is upregulated in LUAD tissues and expressed in LUAD cells}

First of all, IHC staining of 92 LUAD tissues and 70 normal tissues showed the existence of ZNF280A in LUAD tissues, and further indicated the higher level of ZNF280A in LUAD tissues relative to normal ones (Fig. 1A, Table 1). Next, we investigated the statistical correlation between ZNF280A and clinical parameters of patients with LUAD. As shown in Table 2 and Table S4, high level of ZNF280A was positively correlated with clinical characteristics including tumor grade $(P$ $=0.001$, Fig. $1 \mathrm{~A})$, pathological stage $(P=0.011)$ and lymphatic metastasis $(P=0.016)$. Moreover, Kaplan-Meier survival analysis showed the correlation between high ZNF280A expression and relatively lower survival rate (Fig. 1B).

ZNF280A knockdown inhibited LUAD development in vitro

Before carrying out in vitro studies, the expression of LUAD cell lines including A549, NCI-H1299, and SPC-A-
1 was verified, among which A549 and NCI-H1299 cells with relatively higher ZNF280A expression were used as cell models. We upregulated ZNF280A expression of A549 and NCI-H1299 using shZNF280A plasmids, evaluated the transfection efficiency by fluorescence imaging (Fig. S1), and assessed the knockdown efficiency by qPCR and western blotting (Fig. 2A), respectively. 3-(4,5-dimethylthiazol-2-yl)-2,5-diphenyltetrazolium bromide (MTT) assay showed that knockdown of ZNF280A significantly inhibited LUAD cell proliferation (Fig. 2B). The results of flow cytometry indicated the enhanced cell apoptosis of shZNF280A cells, which may be resulted from the G2 phase arrest of cell cycle (Fig. 2C, D). Moreover, the expression of a series of apoptosis-related proteins in LUAD cells with or without ZNF280A knockdown was detected, showing the upregulation of Caspase3, Fas, HSP60, IGFBP-6, TNF- $\beta$, TRAILR-1, and TRAILR-2, and downregulation of Bcl-2, CD40, IGF-II, Livin, and Survivin (Fig. S2). Furthermore, the combination of woundhealing assay and Transwell assay indicated the decreased cell migration capacity in shZNF280A cells (Fig. 2E, F). All these results demonstrated the inhibition of LUAD by ZNF280A knockdown, which was consistent with the IHC results.

\section{ZNF280A knockdown inhibited tumor growth of LUAD in vivo}

For in vivo study, subcutaneous xenografts were established through injection of NCI-H1299 cells with or without ZNF280A knockdown. The observations of in vivo fluorescent imaging and tumor growth curve showed that shCtrl tumors grew more rapidly than the shZNF280A tumors (Fig. 3A, B). The larger size and weight of shCtrl tumors were also verified after collecting xenografts after sacrificing mice (Fig. 3C, D). Consistently, ZNF280A insufficiency induced the lower expression of $\mathrm{Ki67}$, as well as lower proliferative activity, in shZNF280A tumors (Fig. 3E). The outcomes of in vivo experiments were also in agreement with the previously mentioned results, indicating the inhibition of LUAD by ZNF280A knockdown.

\section{The potential of EIF3C as the downstream of ZNF280A in the regulation of LUAD}

For further insight into the mechanism by which ZNF280A regulates LUAD, microarray analysis of NCIH1299 cells with or without ZNF280A identified 6250 differentially expressed genes (DEGs), including 2735 upregulated ones and 3515 downregulated ones (Fig. 4A, S3A, B). Accordingly, the enrichment of all DEGs in canonical signaling pathways and disease/function was interpreted by IPA, showing EIF2 signaling as one of the most enriched pathways and cancer as the most enriched disease (Fig. S3C, D). Subsequently, a variety of top- 


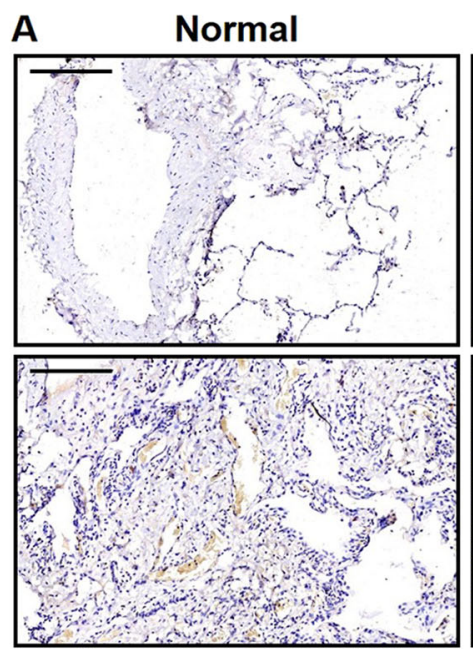

B

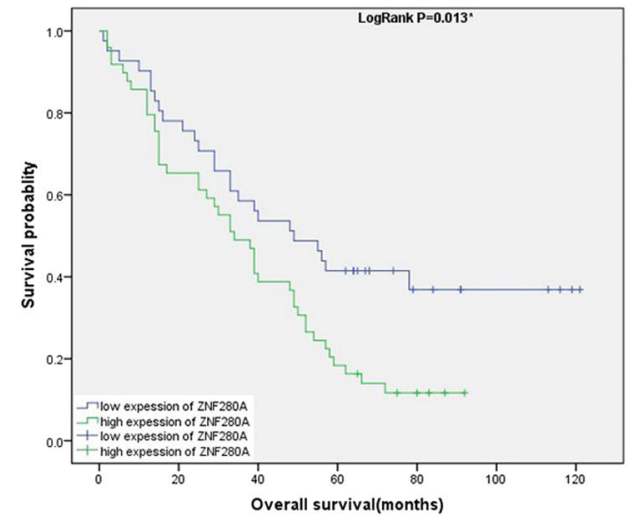

Tumor (Grade II)
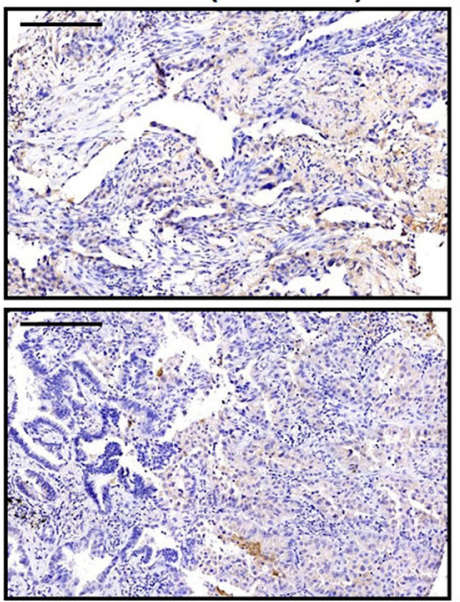

\section{Tumor (Grade III)}

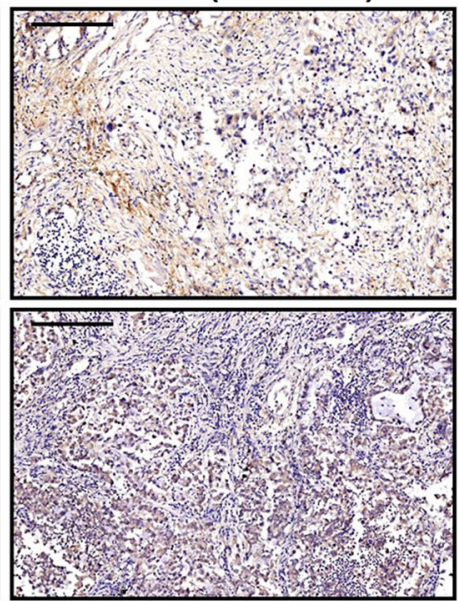

C

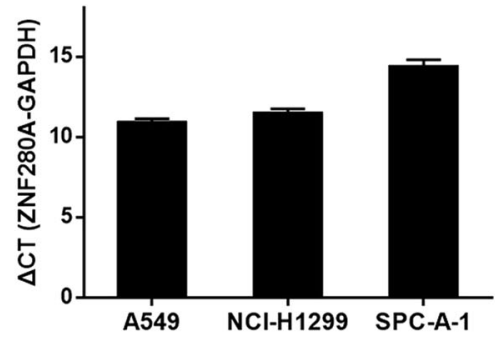

Fig. 1 ZNF280A was upregulated in LUAD tissues and expressed in LUAD cells. A The expression level of ZNF280A was detected by IHC analysis in LUAD tissues and normal tissues (Scale bar $=50 \mu \mathrm{m}$ ). B The Kaplan-Meier survival analysis showed a significant association between ZNF280A high expression and shorter survival period of LUAD patients. C The mRNA expression of ZNF280A in A549, NCI-H1299, and SPC-A-1 cell lines was detected by $\mathrm{qPCR}$. Data were shown as mean $\pm \mathrm{SD}$. ${ }^{*} P<0.05,{ }^{*} P<0.01,{ }^{* * *} P<0.001$.

Table 1 Expression patterns of ZNF280A in lung cancer tissues and normal tissues revealed in immunohistochemistry analysis.

\begin{tabular}{llllll}
\hline \multirow{2}{*}{ ZNF280A expression } & \multicolumn{2}{l}{ Tumor tissue } & & \multicolumn{2}{l}{ Normal tissue } \\
\cline { 2 - 3 } \cline { 5 - 6 } \cline { 5 - 6 } & Cases & Percentage & & Cases & Percentage \\
\hline Low & 42 & $45.7 \%$ & & 70 & $100 \%$ \\
High & 50 & $54.3 \%$ & & 0 & $/$ \\
\hline
\end{tabular}

$P<0.001$.

ranked DEGs was subjected to qPCR and western blotting for assessing the difference of expression induced by ZNF280A (Fig. 4B, C). Further combining the molecular interaction network constructed centered by ZNF280A and based on all above results, EIF3C was regarded as the most promising downstream of ZNF280A (Fig. 4D). As expected, IHC analysis revealed the upregulation of EIF3C in LUAD tissues (Fig. 4E).

\section{Knockdown of EIF3C blocked development of LUAD in vitro}

Using similar strategy with the above-mentioned in vitro study, lentivirus expressing the most efficient EIF3C-targeting shRNA was successfully transfected into NCI-H1299 cells to conduct loss-of-function study of EIF3C (Fig. S4). As anticipated, upon the downregulation of endogenous EIF3C expression in NCI-H1299 cells (Fig. 5A, B), Celigo cell counting assay showed the inhibited cell proliferation (Fig. 5C); colony formation assay indicated the less-formed colonies (Fig. 5D); flow cytometry demonstrated an 8.5-fold elevation of cell apoptosis rate (Fig. 5E); wound-healing and Transwell assays suggested the suppressed migration capability (Fig. 5F, G). The similar effects of ZNF280A knockdown and EIF3C 
Table 2 Relationship between ZNF280A expression and tumor characteristics in patients with lung cancer.

\begin{tabular}{|c|c|c|c|c|}
\hline \multirow[t]{2}{*}{ Features } & \multirow[t]{2}{*}{ No. of patients } & \multicolumn{2}{|c|}{$\begin{array}{l}\text { ZNF280A } \\
\text { expression }\end{array}$} & \multirow[t]{2}{*}{$P$ value } \\
\hline & & low & high & \\
\hline All patients & 92 & 42 & 50 & \\
\hline Age (years) & & & & 0.096 \\
\hline$\leq 61$ & 46 & 17 & 29 & \\
\hline$>61$ & 46 & 25 & 21 & \\
\hline Gender & & & & 0.169 \\
\hline Male & 51 & 20 & 31 & \\
\hline Female & 41 & 22 & 19 & \\
\hline Tumor size & & & & 0.077 \\
\hline$<4 \mathrm{~cm}$ & 39 & 22 & 17 & \\
\hline$\geq 4 \mathrm{~cm}$ & 53 & 20 & 33 & \\
\hline Lymph node-positive & & & & 0.006 \\
\hline$<1$ & 38 & 23 & 15 & \\
\hline$\geq 1$ & 51 & 16 & 35 & \\
\hline Grade & & & & 0.001 \\
\hline I & 3 & 3 & 0 & \\
\hline$\|$ & 61 & 33 & 28 & \\
\hline III & 28 & 6 & 22 & \\
\hline Stage & & & & 0.011 \\
\hline 1 & 27 & 18 & 9 & \\
\hline 2 & 17 & 7 & 10 & \\
\hline 3 & 42 & 13 & 29 & \\
\hline 4 & 1 & 1 & 0 & \\
\hline TInfiltrate & & & & 0.038 \\
\hline T1 & 19 & 13 & 6 & \\
\hline $\mathrm{T} 2$ & 51 & 21 & 30 & \\
\hline T3 & 16 & 7 & 9 & \\
\hline T4 & 6 & 1 & 5 & \\
\hline Lymphatic metastasis (N) & & & & 0.016 \\
\hline No & 38 & 23 & 15 & \\
\hline N1 & 16 & 6 & 10 & \\
\hline N2 & 16 & 2 & 14 & \\
\hline N3 & 4 & 3 & 1 & \\
\hline Expession of EGFR (FISH) & & & & 0.944 \\
\hline Negative & 72 & 34 & 38 & \\
\hline Positive & 13 & 6 & 7 & \\
\hline
\end{tabular}

EGFR epidermal growth factor receptor, FISH fluorescence in situ hybridization. knockdown implied that ZNF280A could be a positive regulator of EIF3C, thus promoting LUAD.

\section{EIF3C knockdown alleviated ZNF280A overexpression induced promotion of LUAD}

In order to further clarify the effects of ZNF280A/EIF3C axis in the regulation of LUAD, ZNF280A overexpression plasmids were used alone for constructing ZNF280Aoverexpressed cell group (ZNF280A group, Fig. S5A for transfection efficiency, Fig. S5B, C for overexpression efficiency), or together with shEIF3C for constructing cell group with simultaneous ZNF280A overexpression and EIF3C knockdown (ZNF280A + shEIF3C group, Fig. S6A for transfection efficiency, Fig. S6B, C for expression detection). As shown in Fig. 6, except for the unexpected regulation of cell apoptosis, ZNF280A overexpression promoted the development of LUAD through accelerating cell proliferation (Fig. 6A), enhancing colony formation (Fig. 6B) and restraining cell migration (Fig. 6D, E). On the other hand, the overall inhibitory effects of cell growth and colony formation (Fig. 6A, B), increase of cell apoptosis (Fig. 6C) and suppression of cell migration (Fig. 6D, E) declared that the ZNF280A expression induced influence on LUAD development could be attenuated or reversed by EIF3C, highlighting the role of ZNF280A/EIF3C axis in LUAD.

\section{Discussion}

The occurrence of LUAD is a slow and long-term complex process involving multi-factors, multi-gene regulation, and network imbalance, which ultimately leads to cell canceration. Although people's understanding of the pathogenesis of lung cancer is not complete, it is not completely unrecognizable. The occurrence and development of tumors mostly result from a series of abnormalities at the molecular level, including mutations or amplification of some genes, abnormal activation or inactivation of signaling pathways, and imbalances in the homeostasis of the cellular environment ${ }^{15}$. A large number of molecules and related signaling pathways in cancer cells have crucial roles in the biological phenotype of LUAD $^{4}$. Therefore, in recent years, more and more studies have focused on exploring the genes related to the proliferation and migration of LUAD and elucidating their regulatory mechanisms, so as to promote the understanding of the molecular mechanism of the occurrence and development of LUAD ${ }^{16,17}$.

Herein, we recognize ZNF280A, a zinc finger protein with $\mathrm{C} 2 \mathrm{H} 2$ domain, as a potential tumor promotor and prognostic indicator in LUAD. High expression of ZNF280A in LUAD tumor tissues was significantly correlated with the advanced grade of tumors and could forecast relatively shorter survival period. The 


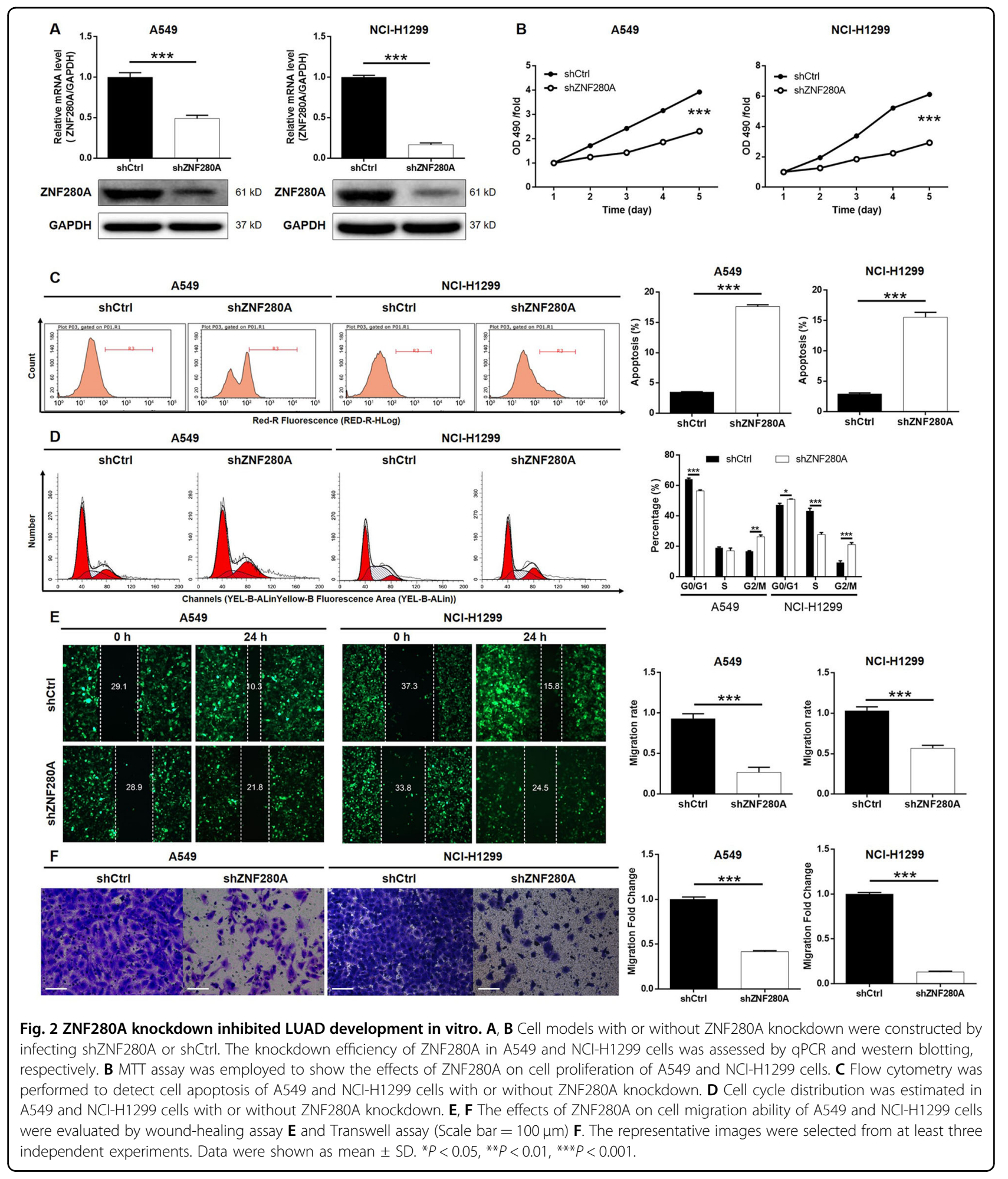

interference of endogenous expression of ZNF280A in LUAD cells suppresses cell growth and induces cell apoptosis through arresting cell cycle in G2 phase. LUAD cells with lower expression of ZNF280A implanted in nude mice also showed significantly decreased in vivo growth. Moreover, ZNF280A knockdown was also expounded to influence migratory capacity of LUAD cells, highlighting its potential role in tumor metastasis. On the other hand, ectopic expression induces converse effects on malignant phenotype of LUAD cells. 


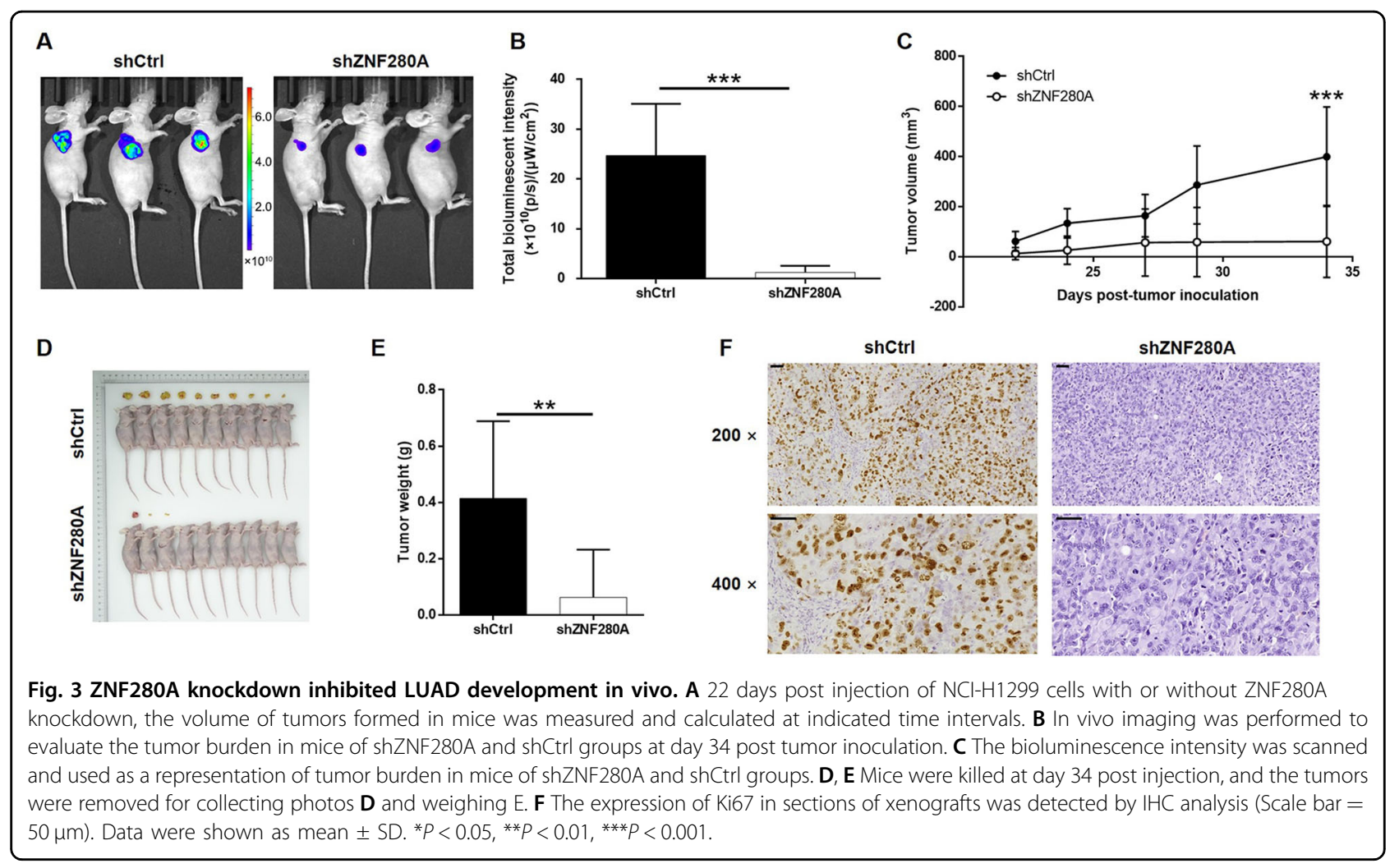

Zinc finger protein refers to a class of protein that contains short, stable, self-folding "finger" structures by binding zinc ions. Owing to its own structural characteristics, it can selectively combine with specific target structures, making zinc finger proteins a critical participant in gene expression regulation, cell differentiation, embryonic development and other life processes ${ }^{18}$. According to the difference of conserved domains, zinc finger proteins can be divided into Cys2/His2-type (C2H2), C3H, C3HC4, C2HC5, C4HC3, C2HC, C4, C6, and $\mathrm{C} 8$ subfamilies ${ }^{19}$. Among them, $\mathrm{C} 2 \mathrm{H} 2$-type zinc finger proteins have been identified as critical regulators in the development and progression of various human cancers. For example, Jen et al. ${ }^{20}$ identified C2H2-type zinc finger protein ZNF322A as a transcriptional inhibitor of c-Myc, thus maintaining lung cancer stem cell-like properties by altering metabolism towards oxidative phosphorylation. Zhang et al. ${ }^{21}$ found that ZNF687 with $\mathrm{C} 2 \mathrm{H} 2$ domain could enhance tumor development and promotes recurrence of hepatocellular carcinoma through transcriptional regulating BMI1, OCT4, and NANOG. On the other hand, although ZNF280A contains two consecutive C2H2-type zinc finger domain transcription factors, there are few studies on its tumor biology. The comprehensive high-resolution whole-genome analysis revealed that ZNF280A is homozygous for deletion in mantle cell lymphoma ${ }^{14}$. Wang et al. ${ }^{22}$ reported the tumor promotion effects of ZNF280A in the development of colorectal cancer, which is resulted from the activation of Hippo signaling.

In order to explore the downstream of ZNF280A in the regulation of LUAD development, gene expression profiling of LUAD cells with or without ZNF280A was obtained, based on which bioinformatics analysis was performed. It was thus deduced that ZNF280A may act as a tumor driver in LUAD by influencing EIF3C expression. A "rescue" test on phenotype regulation of ZNF280A together with EIF3C knockdown revealed EIF3C as a essential mediator in the ZNF280A-induced promotion of LUAD.

The process of eukaryotic translation can be divided into four steps: initiation, extension, termination, and recycling. Translation regulation is mainly realized in the initial stage, which is regulated by 12 known eukaryotic initiation factors (eIFs), which is the speed limiting step in the whole process. In the past decades, the functions of eIFs in the development and progression of malignant tumors have attracted more and more attention ${ }^{23}$. For example, a recent report demonstrated that treatment of $\mathrm{ER}^{+}$breast cancer and KRAS-mutant NSCLC cells with eIF4A inhibitor could inhibit cell cycle feedback response and drug-resistance to CDK4/6 inhibitor treatment ${ }^{24}$. Moreover, the role of eIFs such as eIF6 in lung cancer was also revealed to some extent ${ }^{25}$. eIF3 is the largest and most complex eukaryotic translation initiation factor. It is one of the central factors in 


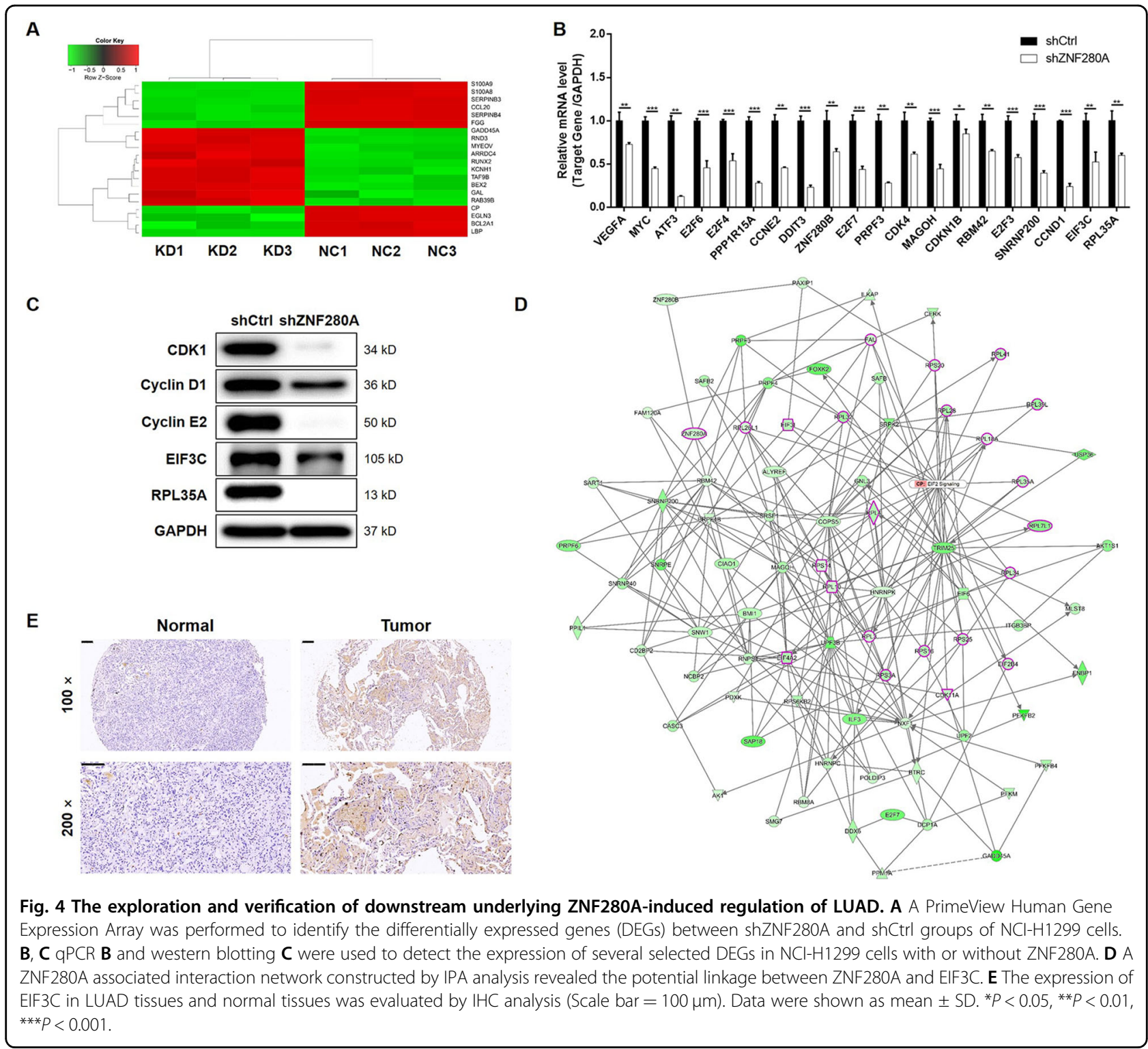

the protein translation initiation complex that interact with other translation initiation factors. At present, it is well-known that eIF3 consists of 13 subunits, which is named eIF3a to eIF3m. Research on eIF3 is mostly concentrated in malignant tumors. In recent years, studies have found that some eIFs subunits are abnormally expressed in tumor cells, and the abnormal expression of these subunits is related to the malignant behavior of tumors ${ }^{26}$. It has been illustrated that some subtypes of eIF3 can affect the prognosis of tumor patients by affecting the proliferation, activation, and apoptosis of tumor cells ${ }^{27}$. For example, EIF3A, the largest subunit of EIF3 family, has been found to be abnormally overexpressed in a variety of malignant tumors, which may be a promising therapeutic target for the design of anti-cancer drugs ${ }^{28}$. EIF3C was also demonstrated to participate in the regulation of human cancers such as ovarian cancer ${ }^{29,30}$, renal cell carcinoma $^{31}$, osteosarcoma ${ }^{32}$, and cervical cancer ${ }^{33}$.
In conclusion, a novel LUAD tumor promotor, ZNF280A, was identified. Upregulation of ZNF280A in LUAD was associated with higher level of malignancy and poorer prognosis. Knockdown of ZNF280A inhibits LUAD development in vitro and in vivo. The mechanistic study proposed that ZNF280A may promote LUAD progression through EIF3C. Generally speaking, ZNF280A/EIF3C axis has a crucial role in LUAD, which may be promising targets for LUAD treatment.

\section{Materials and methods}

Cell lines and cell transfection

A549 and NCI-H1299 cells were purchased from the Cell Bank of Type Culture Collection of Chinese Academy 


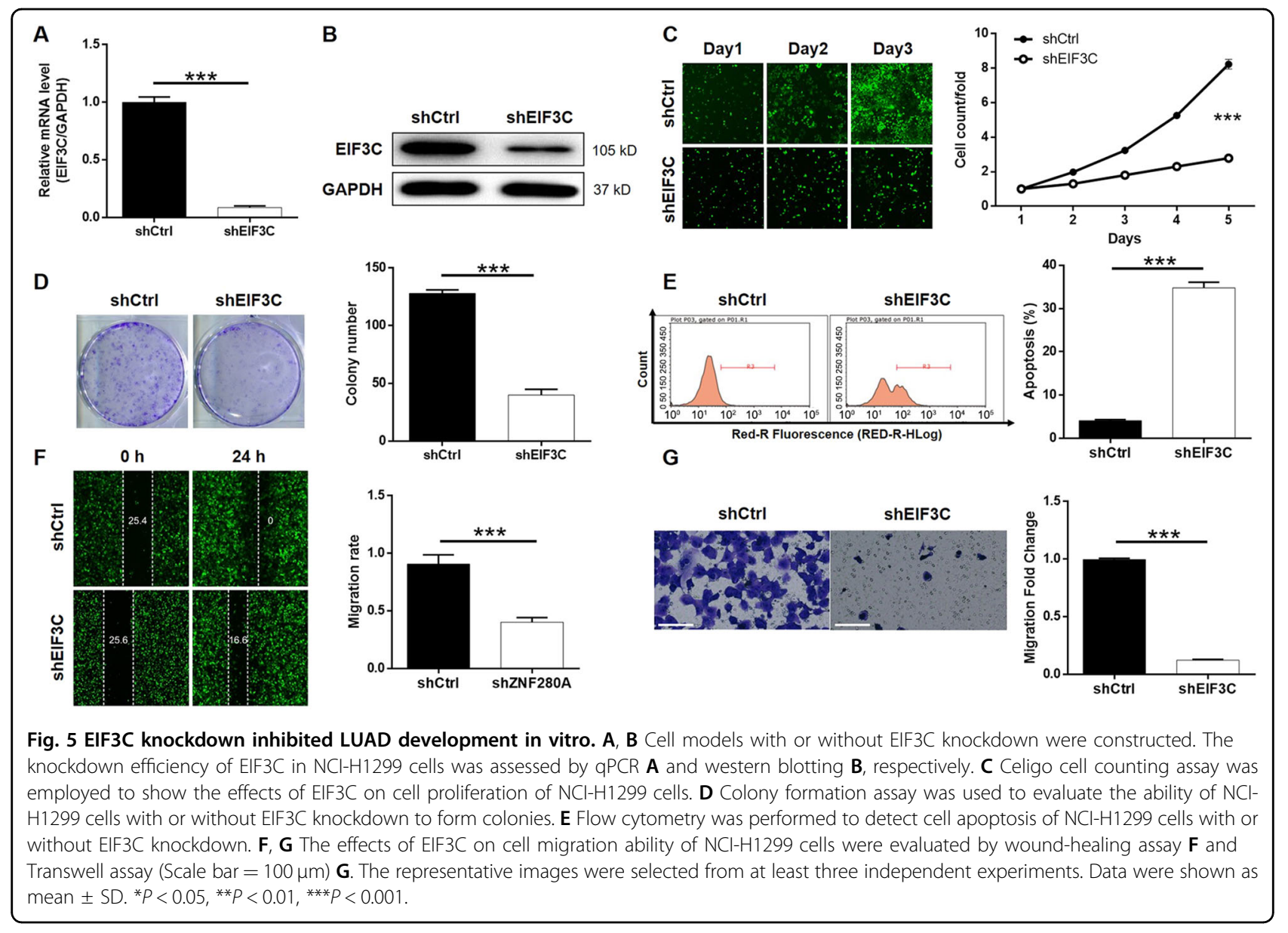

of Sciences and NCI-H1299 were cultured in RPMI-1640 medium (Gibco) with $10 \%$ fetal bovine serum (FBS) and A549 was maintained in McCoy's 5A Medium with $10 \%$ FBS. All cells were cultured in a humidified cell culture incubator at $37^{\circ} \mathrm{C}$ under $5 \% \mathrm{CO}_{2}$ with culture medium changed every $72 \mathrm{~h}$.

For stable gene expressing, lipofectamine RNAimax (Cat. \#13778075, Thermo fish) were used for cell A549 and NCIH1299 transfection with lentiviral plasmids collected. Cells were harvested after $72 \mathrm{~h}$ culturing, and cell infection efficiency was valued with LV-shCtrl cells as control.

\section{IHC staining}

Human lung cancer and para-normal tissue chip (Cat. \#HLugA180Su05, Shanghai Outdo Biotech Company) was used and patients' information was collected. For IHC staining, deparaffinized and rehydrated tissue sections were blocked and incubated with primary antibody ZNF280A (Cat. \#bs-12839R, BIOSS) and followed incubated by secondary antibody. DAB color was developed with diaminobenzene and hematoxylin. Slides were pictured with microscopic and viewed with ImageScope and CaseViewer. All slides were examined randomly by two independent pathologists and IHC outcomes were determined by staining percentage and intensity scores. Staining percentage scores were classified as: 1 (1-24\%), 2 (25-49\%), 3 (50-74\%), and 4 (75\%-100\%). Staining intensity were scored 0 (Signalless color) to 3 (light yellow, brown and dark brown). Antibodies used in IHC were listed in Table S1.

\section{RNA interference and plasmids packaging}

shRNA sequences targeting human ZNF280A and EIF3C gene were designed and cDNAs were synthesized by Shanghai Yibeirui Bioscienceres, Co., Ltd. and subsequently cloned into luciferase-labeled BR-V-108 vector. In addition, ZNF280A was amplified and cloned into the BRV112 vector after double digestion by BamHI and AgeI, and sequenced. Lentiviral particles were collected, following co-transfection using pHelper 1.0 and pHelper 2.0 vector for plasmids packaging. The sequences used were listed in Table S2.

\section{RNA extraction and RT-qPCR}

After $72 \mathrm{~h}$ for ZNF280A and/or EIF3C RNA expressing, A549 and NCI-H1299 cells in triplicate were fully lysed 


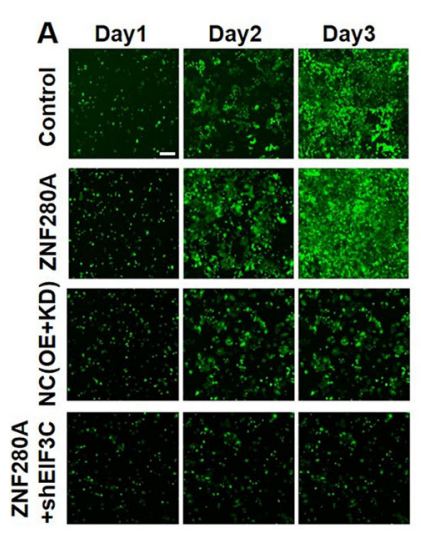

B

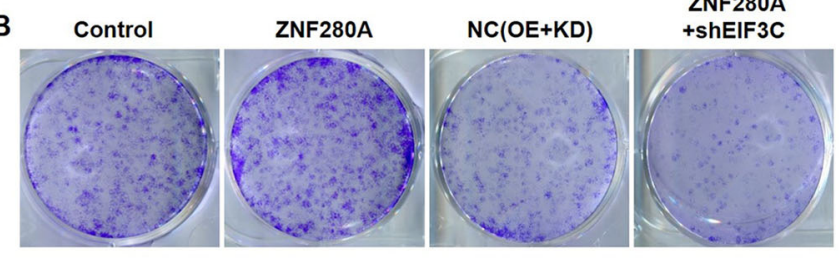

C

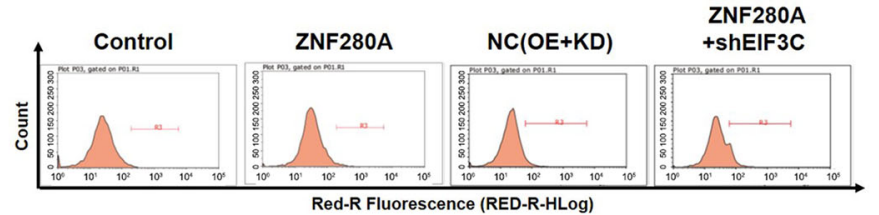

D Control

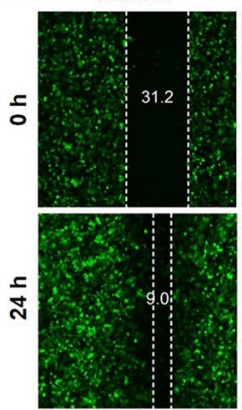

E
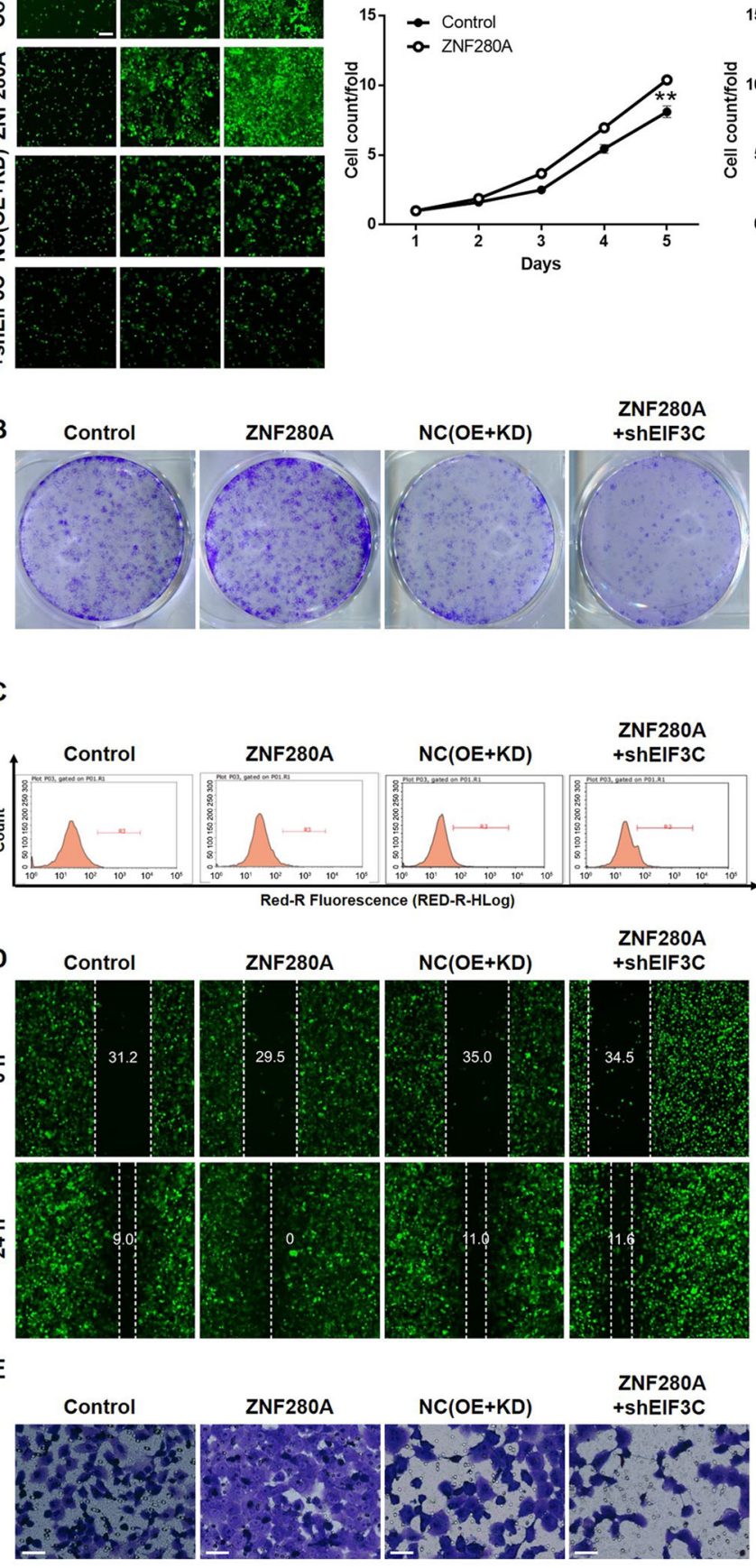

NC(OE+KD)

ZNF280A
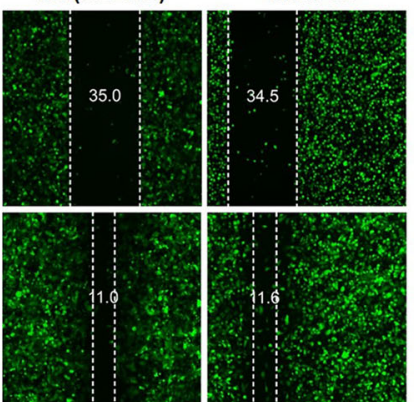
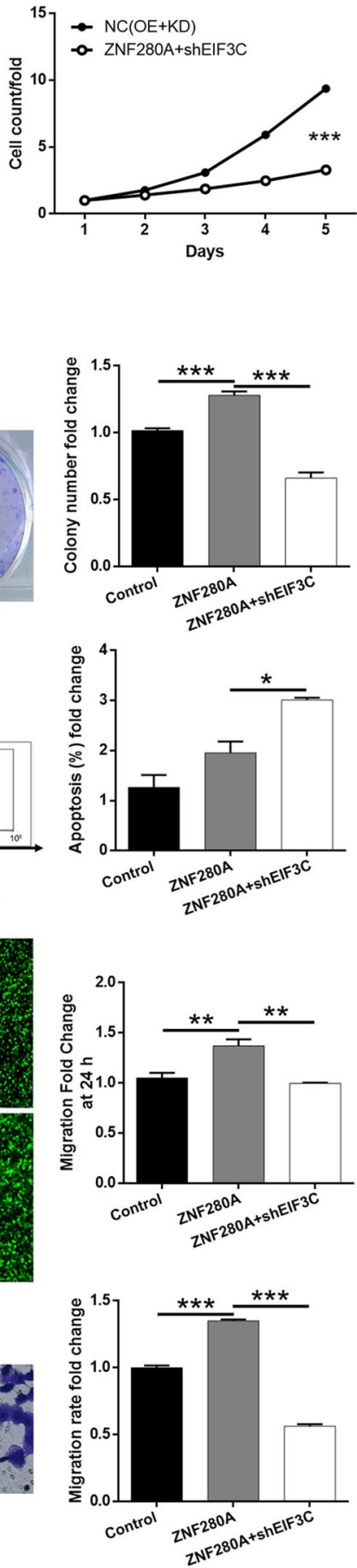

Fig. 6 Knockdown of EIF3C attenuated the effects of LUAD cells by ZNF280A overexpression. NCI-H1299 cells transfected with Control plasmids, ZNF280A overexpression plasmids, NC(OE + KD), and simultaneous ZNF280A overexpression plasmids and shEIF3C were subjected to the detection of cell proliferation by Celigo cell counting assay (Scale bar $=400 \mu \mathrm{m}) \mathbf{A}$, colony formation B, cell apoptosis by flow cytometry C, cell migration by wound-healing assay D and cell migration by Transwell assay (Scale bar $=50 \mu \mathrm{m}) \mathbf{E}$. The representative images were selected from at least three independent experiments. Data were shown as mean $\pm \mathrm{SD}$. ${ }^{*} P<0.05$, ${ }^{* *} P<0.01,{ }^{* * *} P<0.001$. 
and total RNA was extracted using TRIzol reagent (Sigma). The RNA quality was evaluated by Nanodrop 2000/2000C spectrophotometer (Thermo Fisher Scientific). cDNA was reversely transcribed from RNA using Promega M-MLV Kit (Promega) and qPCR was performed with SYBR Green mastermixs Kit (Vazyme) by applying Biosystems 7500 Sequence Detection system. Glyceraldehyde 3-phosphate dehydrogenase (GAPDH) was acted as inner control, and the primers used for the PCR reaction were showed in Table S3. The relative quantitative analysis in gene expression data were analyzed by the $2^{-\Delta \Delta \mathrm{Ct}}$ method.

\section{Western blotting, co-immunoprecipitation, and human apoptosis antibody array}

Cells were lysed in ice-cold radioimmunoprecipitation assay buffer (Millipore), and the protein were collected and the concentration was detected by a BCA Protein Assay Kit (HyClone-Pierce). Protein samples $(20 \mu \mathrm{g}$ per lane) were separated by $10 \%$ sodium dodecyl sulfate polyacrylamide gel electrophoresis (Invitrogen), and transferred onto polyvinylidene difluoride membranes at $4{ }^{\circ} \mathrm{C}$. The membranes were blocked with tris-buffered saline tween-20 solution of $5 \%$ degreased milk at room temperature for $1 \mathrm{~h}$ and incubated with primary antibodies and GAPDH antibodies at $4{ }^{\circ} \mathrm{C}$ overnight. Then the membranes were incubated with secondary antibody HRP goat anti-rabbit IgG for $2 \mathrm{~h}$ at room temperature. The blots were visualized by enhanced chemiluminescence (Amersham).

For Human Apoptosis Antibody Array, briefly, $20 \mu \mathrm{g}$ total proteins were cultured with the antibody-coated array membranes and then continuing incubated with HRP linked Streptavidin conjugate.

All the antibodies used in western blotting were listed in Table S1.

\section{Cell proliferation analysis}

The cell viability was determined by MTT assay, briefly, transfected A549 and NCI-H1299 cells were stained with MTT reagent $(5 \mathrm{mg} / \mathrm{mL}$, GenView) and Formazan was dissolved by DMSO solution. The absorbance values at $490 \mathrm{~nm}$ were measured by microplate reader (Tecan) and the reference wavelength was $570 \mathrm{~nm}$.

Cell proliferation rate was analyzed by Celigo cell counting assay. In brief, targeting cells were seeded at a 96-well plate with 2000 cells per well. The plate was continuously detected by Celigo (Nexcelom) for 5 days at the same time.

For colony formation assay, cells in the logarithmic growth phase were seeded into 6-well plates in triplicate and further cultured for 8 days. Cell clones were fixed with $4 \%$ paraformaldehyde and stained with Giemsa. Then clones were photographed under a fluorescence microscope (Olympus) and colony number (clone contains $>50$ cells) was counted.

\section{Cell apoptosis and cells cycle assay}

The flow cytometric methods of identifying apoptotic cells was applied using Annexin V-APC Apoptosis kit (Cat. \#88-8007, eBioscience). For cells cycle assay, cells were stained with $1 \mathrm{~mL}$ propidium iodide (PI) staining solution $(40 \times$ PI, $2 \mathrm{mg} / \mathrm{mL}$ : $100 \times$ RNase, $10 \mathrm{mg} / \mathrm{mL}: 1 \times$ $\mathrm{PBS}=25: 10: 1000$ ). FACScan and FlowJo 7.6.1 (Ashland) was used for analyze. Cell apoptosis was measured and the percentage of the cells in G0-G1, S, and G2-M phase were counted and compared.

\section{Cell migration assays}

In order to analyze the migration ability of transfected cells in our research, wound-healing assay and transwell assay were performed. For wound-healing assay, lentivirus transfected A549 and NCI-H1299 cells $\left(5 \times 10^{4}\right.$ cells/well) were plated into 96-well plates for culturing. Scratches were made by a 96 wounding replicator (VP scientific). Photographs were taken by a fluorescence microscope at $0 \mathrm{~h}, 8 \mathrm{~h}$, and $24 \mathrm{~h}$ and cell locations were recorded, respectively. Cell migration rates of each cell group were calculated. In transwell assay, cells were seeded into a 24well plate in the upper chambers, and medium supplemented with 30\% FBS was added into the lower chambers. Cells were fixed with $4 \%$ formaldehyde and stained by Giemsa and the migration ability of cells was analyzed.

\section{Microarray analysis}

Total RNA from NCI-H1299-shCtrl and NCI-H1299shZNF280A cells was extracted using TRIzol. RNA quantity and quality were assessed with a Thermo Nanodrop $2000(1.7<$ A260/A280 < 2.2, Thermo Fisher Scientific). Affymetrix PrimeView Human Gene Expression Arrays (Thermo Fisher Scientific) were used for microarray analysis to obtain gene expression profiles according to the manufacturer's instructions. Significantly DEGs were selected based on $P<0.05$ and |Fold Change | $>1$.3. KEGG pathway enrichment analysis was performed for all significant DEGs.

\section{Animal experiments}

All animal studies were approved by Ethics committee of Peking Union Medical College Hospital. Female BALB/ c nude mice were purchased from Shanghai Lingchang Experimental Animals Co., Ltd. For tumorigenicity, $5 \times$ $10^{6}$ lentivirus (shCtrl or shZNF280A) transfected NCIH1299 cells were subcutaneously injected into each mouse (4-week-old, $n=10$ per group). Mice's weight and tumor sizes were recorded two times per week and the volume of tumor was calculated as $\pi / 6 \times L \times W^{2}(W$, 
width at the widest point; $L$, perpendicular width). Finally, the tumor burden was assessed by bioluminescence imaging with non-invasive IVIS Spectrum Imaging System (Perkin Elmer). Mice were killed then tumors were extracted and imaged.

\section{Ki67 immunostaining assay}

Mice tumor sections were fixed in $4 \%$ paraformaldehyde. Paraffin embedded $5 \mu \mathrm{m}$ sections were made for hematoxylin and eosin and IHC staining. We added citric acid buffer for antigen retrieval at $120^{\circ} \mathrm{C}$. Sections were blocked using PBS- $\mathrm{H}_{2} \mathrm{O}_{2}$ with $0.1 \%$ Tween-20. Ki67 antibody was added for incubating at $4{ }^{\circ} \mathrm{C}$ overnight and then secondary antibodies were added as well. DAB color was developed with diaminobenzene and hematoxylin. Stained slides were pictured with a microscopic.

\section{Statistical analyses}

Each experiment was repeated three times and the data were shown as mean $\pm \mathrm{SD}$. Categorical variables were expressed as percentages. The significance between groups was determined using the two-tailed Student's $t$ test or one-way analysis of variance. Relationship between ZNF280A expression and tumor characteristics in lung cancer patients with was analyzed using Mann-Whitney $U$ analysis and Spearman grade correlation analysis. Statistical significance was calculated by SPSS 22.0 (IBM) and $P$ value $<0.05$ was considered statistically significant. Graphs were made using GraphPad Prism 6.01 (Graphpad Software).

\section{Acknowledgements}

This study was financially supported by CAMS Initiative for Innovative Medicine (CAMS-2017-12M-4-002), National Natural Science Foundation of China (no. 61435001), CAMS Innovation Fund for Medical Sciences (no. 2016-12M-1-001), National Natural Science Foundation of China (no. 81472785; no. 61435001) and CAMS Innovation Fund for Medical Sciences (no. 2016-12M-1-001).

\section{Author contributions}

Y.W. designed and supervised the program. H.L. and Y.Q. conducted the in vitro and in vivo experiments. N.Z. performed all the data analysis and bioinformatics analysis. The manuscript was produced by D.M., H.L., and Y.Q. which was checked by Y.W. All the authors approved the submission of this manuscript.

\section{Conflict of interest}

The authors declare that they have no conflict of interest.

\section{Publisher's note}

Springer Nature remains neutral with regard to jurisdictional claims in published maps and institutional affiliations.

Supplementary Information accompanies this paper at (https://doi.org/ 10.1038/s41419-020-03309-9).

Received: 12 June 2020 Revised: 12 October 2020 Accepted: 14 October 2020

Published online: 04 January 2021

\section{References}

1. Siegel, R. L., Miller, K. D. \& Jemal, A. Cancer statistics, 2020. CA Cancer J. Clin. 70, 7-30 (2020).

2. Bray, F. et al. Global cancer statistics 2018: GLOBOCAN estimates of incidence and mortality worldwide for 36 cancers in 185 countries. CA Cancer J. Clin. 68, 394-424 (2018).

3. Ettinger, D. S. et al. Non-small cell lung cancer, version 5.2017, NCCN clinical practice guidelines in oncology. J. Natl. Compr. Canc. Ne. 15, 504-535 (2017).

4. Petersen, I. \& Warth, A. Lung cancer: developments, concepts, and specific aspects of the new WHO classification. J. Cancer Res. Clin. 142, 895-904 (2016).

5. Torre, L. A., Siegel, R. L. \& Jemal, A. Lung cancer statistics. Adv. Exp. Med. Biol. 893, 1-19 (2016)

6. Barr Kumarakulasinghe, N., Zanwijk, N. V. \& Soo, R. A. Molecular targeted therapy in the treatment of advanced stage non-small cell lung cancer (NSCLC). Respirology 20, 370-378 (2015).

7. Walder, D. \& O'Brien, M. Looking back and to the future: are we improving 'cure' in non-small cell lung cancer? Eur. J. Cancer 75, 192-194 (2017).

8. Rosell, R. \& Karachaliou, N. Adjuvant therapy for resected EGFR-mutant nonsmall-cell lung cancer. Lancet Oncol. 19, e126 (2018).

9. Wang, J. et al. circRNA-002178 act as a ceRNA to promote PDL1/PD1 expression in lung adenocarcinoma. Cell Death Dis. 11, 32 (2020).

10. Cai, Y., Sheng, Z., Chen, Y. \& Wang, J. LncRNA HMMR-AS1 promotes proliferation and metastasis of lung adenocarcinoma by regulating MiR-138/sirt6 axis. Aging 11, 3041-3054 (2019).

11. Brayer, K. J. \& Segal, D. J. Keep your fingers off my DNA: protein-protein interactions mediated by $\mathrm{C} 2 \mathrm{H} 2$ zinc finger domains. Cell Biochem. Biophys. 50, 111-131 (2008).

12. Jen, J. \& Wang, Y. Zinc finger proteins in cancer progression. J. Biomed. Sci. 23 53 (2016).

13. $\mathrm{Wu}, \mathrm{X}$. et al. Zinc finger protein 367 promotes metastasis by inhibiting the Hippo pathway in breast cancer. Oncogene 39, 2568-2582 (2020).

14. Beà, S. et al. Uniparental disomies, homozygous deletions, amplifications, and target genes in mantle cell lymphoma revealed by integrative high-resolution whole-genome profiling. Blood 113, 3059 (2009).

15. Herbst, R. S., Morgensztern, D. \& Boshoff, C. The biology and management of non-small cell lung cancer. Nature 553, 446-454 (2018).

16. Wang, C. et al. RNA-Seq profiling of circular RNA in human lung adenocarcinoma and squamous cell carcinoma. Mol. Cancer 18, 134 (2019).

17. Wei, C. et al. LPCAT1 promotes brain metastasis of lung adenocarcinoma by up-regulating PI3K/AKT/MYC pathway. J. Exp. Clin. Cancer Res. 38, 95 (2019).

18. Hamed, M. Y. \& Arya, G. Zinc finger protein binding to DNA: an energy perspective using molecular dynamics simulation and free energy calculations on mutants of both zinc finger domains and their specific DNA bases. J. Biomol. Struct. Dyn. 34, 919-934 (2016).

19. Han, $\mathrm{G}$. et al. $\mathrm{C} 2 \mathrm{H} 2$ zinc finger proteins: master regulators of abiotic stress responses in plants. Front. Plant Sci. 11, 115 (2020).

20. Jen, J. et al. Oncogenic zinc finger protein ZNF322A promotes stem cell-like properties in lung cancer through transcriptional suppression of c-Myc expression. Cell Death Differ. 26, 1283-1298 (2019).

21. Zhang, T. et al. Overexpression of zinc finger protein 687 enhances tumorigenic capability and promotes recurrence of hepatocellular carcinoma. Oncogenesis 6, e363 (2017).

22. Wang, $X$. et al. ZNF280A promotes proliferation and tumorigenicity via inactivating the hippo-signaling pathway in colorectal cancer. Mol. Ther. Oncolytics 12, 204-213 (2019).

23. Spilka, R., Ernst, C., Mehta, A. K. \& Haybaeck, J. Eukaryotic translation initiation factors in cancer development and progression. Cancer Lett. 340, 9-21 (2013).

24. Kong, T. et al. elF4A inhibitors suppress cell-cycle feedback response and acquired resistance to CDK4/6 inhibition in cancer. Mol. Cancer Ther. 18, 2158 (2019).

25. Gantenbein, N. \& Mitterer, V. Influence of eukaryotic translation initiation factor 6 on non-small cell lung cancer development and progression. Eur. J. Cancer 101, 165-180 (2018).

26. Gomes-Duarte, A., Lacerda, R., Menezes, J. \& Romão, L. elF3: a factor for human health and disease. RNA Biol. 15, 26-34 (2018).

27. Yin, $Y$. et al. The function and clinical significance of elF3 in cancer. Gene $\mathbf{6 7 3}$ 130-133 (2018) 
28. Yin, J. Y., Zhang, J. T., Zhang, W., Zhou, H. H. \& Liu, Z. Q. elF3a: a new anticancer drug target in the elF family. Cancer Lett. 412, 81-87 (2018).

29. Liu, T. et al., The m6A reader YTHDF1 promotes ovarian cancer progression via augmenting EIF3C translation. Nucleic Acids Res. 48, 3816-3831 (2020).

30. Wen, F. et al. Eukaryotic initiation factor 3, subunit $C$ silencing inhibits cell proliferation and promotes apoptosis in human ovarian cancer cells. Biosci. Rep. 39, BSR20191124 (2019).
31. Fan, M. et al. Upregulated expression of elF3C is associated with malignant behavior in renal cell carcinoma. Int. J. Oncol. 55, 1385-1395 (2019).

32. Gao, W. et al. Knockdown of EIF3C promotes human U-2OS cells apoptosis through increased CAS P3/7 and Chk1/2 by upregulating SAPKJJNK. Oncotargets Ther. 12, 1225-1235 (2019).

33. $\mathrm{Hu}, \mathrm{C}$. et al. Overexpressed circ_0067934 acts as an oncogene to facilitate cervical cancer progression via the miR-545/EIF3C axis. J. Cell. Physiol. 234, 9225-9232 (2018) 